\title{
Novel strategy of a method to cure from the cancer
}

\author{
Adnan Yousif Rojeab \\ Electrical and Electronic Engineering Department, The London College UCK, London, U.K.
}

\section{Email address:}

rojeab@btinternet.com

\section{To cite this article:}

Adnan Yousif Rojeab. Novel Strategy of a Method to Cure from the Cancer. Cancer Research Journal. Vol. 3, No. 1, 2015 , pp. 6-10. doi: $10.11648 /$ j.crj.20150301.12

Abstract: Cancer is a reaction mechanism which is only created to eliminate severe damage and dangerous occur in the cells, when the immune system of the body falls to cure the damage. The action of the cancer is, only, ended when a direct, right and simple treatment method is applied to cure the previous diseases which caused the cancer, but not to treating the cancer itself.

Keywords: Cancer Natural Action, Lengthening the Telomere in Germs and Cancer, History of Old Diseases, New Strategy to Treat the Cancer, Lengthening of the Telomere, Treat Old Diseases before Cancer

\section{Introduction}

Cancer subject is widely known. The importance of this introduction is to explain the broad and significant factors in the cancer creation. The factors are those directly concerning with the main points to be discussed in this hypothesis research.

In this regard, it is relevant to avoid any biological or chemical details, and to concentrate on the points related to the main topic of this paper.

Vital processes such as cell division and cell function are controlled by certain genes, where great damaging in some of these genes can lead to a cell to become cancerous [1].

Accordingly, cancer is a disease characterized, in literature, by the development of abnormal cells that divide uncontrollably and have the ability to infiltrate and destroy normal body tissue. This is because the normal cells, regulator mechanisms have been damaged; when cells keep dividing, while new cells are not required by the body, a tumor forms.

The faulty genes may be inherited or caused by carcinogens (cancer-causing agents), such as radiation, sunlight, tobacco smoking and virus [2].

On the other hand cells are continually exposed to carcinogens, but they rarely become cancerous for some reasons such as: cells can usually repair damages; more than one gene must be damaged before cancer develops; and the body's immune system often destroys any abnormal cells before they are able to multiply enough to form a cancerous tumor.

If this process fails, then, cancer developing is a matter of severe damaging to specific genes.
Cancer is a group of many different diseases [3], it depends on the causing factor and the area (tissue) been affected.

The target of this hypothesis is to rise a vital guide strategy leading to considerable decrease in the number of sufferers from the cancer.

\section{Telomere and the Effect of its Length}

Telomeres are specialized composed of DNA - protein structure, where, they are positioned at the terminals of each chromosome. Their function is to protect ends of the chromosome from damage, and prevent the chromosome from fusion into rings or binding with other chromosomes in the nucleus [4].

Telomeres of most cells are shortened, (in each DNA replication), which is for each cell division where this is causing the cell to die after certain number of divisions. While the telomeres of cancer cells are extended by the control of enzyme telomerase, which is leading to that the cancer cells will form, over time, a tumor, and the cancer cells accounted to be immortal.

The shortening of the telomere is the result of the magnetic induction during DNA replication, which is governed by the magnetic phenomenon [5]. Therefore, the action of magnetic field, in turn, may be applied in such a way to help for treatment and then to cure from the cancer.

Concerning with length, telomeres in germ cells are different from those in somatic cells, where:

(a) Somatic cells are the great majority of the body's cells. After the age of the early fetus development, genes producing telomerase are inactive in the 
somatic cells, for the whole of the life.

When the replication process of DNA is taking place, the new DNA is left with shortening telomeres, comparing with those in the parental ones [4]. Inactive telomerase will not be able to lengthening these telomeres. This is an essential process to save the kind size.

(b) Germ cells are sperm and egg themselves described from progenitor cells. Telomerase, in turn, is highly active in germ cells line, extended sperm telomeres are brought to the zygote during fertilization, and serve as a species-specific zero - time length mark for the subsequent cell division in progeny. This is essential for the human and other kinds.

When there is no mechanism for replicating lost telomere, then all organisms with linier chromosomes (eukaryotes) would be condemned to quick extinction in their reproductive tissues.

Genes, produce telomerase, are active through the whole life in few special groups of cells such as antibodies producing immune cells, cells that replenish the gut lining and sperm producing cells.

\section{Causes and Consequences}

There are great number of factors which are producing cancerous cells [1], where they can be accounted as a complex list. Main factors are [2], environment pollutants, chemical effects, tobacco use, exposure to radiation and /or excessive exposing to sunlight, viruses, biological which is by excess hormones, and other factors which are, generally, accounted as less relevant. These factors are acting to damage genes, and to produce genetic faults within the cells [6].

It is shown that, many types of cancers could be prevented by experiences of many factors such as: non- smoking, providing healthy food, applying physical exercising, minimizing sunlight exposure, and being vaccinated against some infectious diseases.[7]

\section{Methods to Eliminate Cancer}

It is obvious that, cancer is various types, accordingly, there are many methods been applied in aiming to eliminate the cancer. The main methods are:

(a) Surgery therapy: surgery is applied to remove tumor, where it is a local therapy. Also, during surgery, it is usual to remove some normal tissue surrounding the tumor to maximize the chances that all cancerous cells are removed from the body. It has been indicated that remove tumors by surgery may leave cancer cells to remain and grow again.

(b) Chemotherapy: it is a therapy of using drug to eliminate the cancer. Using chemotherapy to stop cancer cells from dividing can be effective in some types of cancers but not the others.

(c) Radiotherapy: it is a therapy of using high energy rays directed to damage cancer cells to eliminate their growing and dividing. The therapy is local, where it, may affect cancer cells only into treated area.

It is found that, medical use of ionizing radiation is a growing source of radiation-induced cancers. Ionizing radiation may be used to treat other cancers, but this may, in some cases, induce a second form of cancer [1].

(d) Biological therapy (or immunotherapy): it is a treatment of using the immune system of the body to fight infection and disease

(e) Hormone therapy: it is a therapy that preventing cancer cells from getting or using the hormones they need.

The therapy concerns with most breast and prostates types of cancer, which depend on hormone to grow. In order to stop hormone production, a method of using drugs is applied, or a surgery has to remove organs produce hormone (such as ovaries or testicles). The therapy affects cells throughout the body.

Therefore, it is clear that, every form of the above treatments has its own side effect, which means it has the affection to harm healthy tissues under those treatment.

\section{Discussion}

It is believed that any attempt method to eliminate the effect of the cancer should, only, be applied by understanding the fundamental reason of its existence. This understanding leads to create a plan of strategy to deal with the cancer.

To apply this concept, it should be concentrated on the original occurrence to create the onset of the cancer.

Accordingly, it is important to arise some practical results gained by researchers on the cancer and by its patients, in order to plan a right and significant strategy to cure from the cancer. These results show that:

A. Cells are continually exposed to carcinogens but they rarely become cancerous. This is because these cells usually repair their damaged genes through the immune system, which often destroys any cause of abnormal cells to form a cancerous tumor.

B. When an acting factor creates cancer in some persons, but it is not created in others. While, a cell may become cancerous, is when certain genes, that control vital processes such as cell division, should become damaged.

C. Strengthening the body by healthy nutrition, physical exercises, no - smoking, out of pollution etc..., then the cancer could be eliminated.

By analytic the above results, some certain points should be looked at, they are:

i. The cancer is not an agent which acts to damage the cells of the body, but it is the result of other factors which are severely affecting the body. It means that the cancer is not originally existed, but its occurrence is associated with the danger applied on the body.

ii. Although cells exposed to carcinogens, but the 
effect, may be, transient when the immune system of the body is able to destroy the dangerous on the cells, as long as the effect of the carcinogen is not severe. Also, it can be assumed that if the body is naturally a healthy one, and it is not affected by any other diseases or damage, so, it is believed that there is no need to worry for a possible existence of the cancer.

iii. The danger process for the onset of the cancer will not exist unless a severe damage is applied against the vital functions which are controlled by the genes. Where great damage or disease, is threatening to dissolve the active function of the genes, (with lack of immune ability to cure).

Accordingly, if the immune system of the body is lacking behind the ability to repair the severe damage of the carcinogen, then there should be a certain reaction to arise to save the body. This reaction is a mechanism tries to substitute both the damaged and dye cells, by healthy and active ones.

The first performing step of this mechanism is to induce the action of enzyme telomerase, where it tends to lengthening the telomeres of the DNA of the healthy and new cells in the affected area [tissue]. The lengthening of the telomere is, believed to be, counted as a vital performance which is to strengthening the structure process of the DNA molecule.

This procedure, which is so called cancer, is aiming to enforcing the function of the telomere and to create new type of cells, while the carcinogens are aiming to destroy the structure of the DNA. This means that, the cancer performance is opposing to that of the carcinogens.

Therefore, the cancer is just created because of the severe effect of the carcinogens factors, but it is practically acting to oppose the action of these factors.

Also, concerning with the matter of the cancer, it is important to realize the inherent processes occur in the body, are:

It can be seen that the action done by the cancer is although creating a tumor but also producing a type of somatic cells similar, [in telomere term], to those very important and fundamental cells, which are the germ cells.

In fact, the existence of tumor does not mean the lengthening of the telomere is always a dangerous matter leads to cause threatening to create a tumor.

The lengthening of the telomere is essential and important process in the germ cells and in other certain tissues of the body. This process, as a fact, is to secure the living kind regenerations, because the telomerase is active in these cells to performing the lengthening of their telomere.

Thus, it can be understood that, the cancer's aiming in this process is similar [analogue] to that of the telomerase enzyme in performing the lengthening of the telomeres, but the issue is that each acts on a certain sort of cells.

It is believed that this realization, may lead to show that:

The vital genes produce the enzyme telomerase, (which is active in germ cells), are similar to those genes which might produce a certain mechanism in somatic cells and leads to cancer, (to be active against the damaging to the vital function of the somatic genes).

This mean that, active genes in somatic cells try to cure their effected cells by creating an action, [a mechanism], to eliminate the sever action of the carcinogen factors.

The points, previously discussed, may indicate that, the onset of the cancer only occurs in order to overcome sever damages affecting the vital processes in cells, while the other circumstances, (such as the immune system of the body and outside therapy treatments), are not able to stop the dangerous damages.

The analysis is an important, to understand the essential strategy for the cancer's existence, and for its performing activity. It is believed that this understanding for the fundamental existence of the cancer will have a great relationship to that strategy when planning to cure the cancer. That is, to find a vital method to stop the onset of the cancer itself.

Also, it may be seen that, the original target for the process of the onset of the cancer is: a performing aims to work for the benefit of the cells of the body, while the side effect is a disaster. Although, the existence of that disaster, may lead to discover a new strategy to cure the cancer.

As a fact, the cancer process is preventing the telomeres from getting shorter, by performing the lengthening at every DNA replication process in the somatic cells, which will lead to create somatic cells to grow in regenerations without limitation, where they will be immortal [do not die].

This is believed to be exactly the target of the cancer process, as long as its aim is to overcome the severe damage which effecting the vital function of the genes.

Accordingly, the process of the cancer to eliminate the severe damage in the cells is performing as that is showing in the following model:

When a severe damage effects the vital function of a cell, a programming code in the cell will be active, in reaction, to oppose that danger damage.

The initial reaction against the damage is, then, to activate the ability of the vital cell's functional in order to eliminate the cause of that damage. This reaction can be done by overcoming both the weak cells and those cells which are lost their performance for vital processes. So the damaged tissue remains with lack of the number of cells.

The process, then, may be continued by trying to substitute these damaged cells by producing an increase number of healthy and active ones. So, it is to secure the continuation of increasing enough number of healthy and active cells in the damaged tissue.

In order to settle this state, lengthening the telomere should be secured throughout the cell regenerations, (for daughter cells), in the affected tissue. This process is accounted as the best way to keep the tissue possesses healthy and active cells by substitute dye cells, and to face future sever damages.

It may be relevant to comment on the natural existence of germ cells and cancerous somatic cells, where to show that:

Lengthening the telomeres of the germ cells is essential, at the same time, they are not representing a sort of tissue of 
cells. It is to say that, there is no way to think that whether there is a tumor or not when the telomeres of these germ cells are always lengthened.

While, concerning with the somatic cells, it is essential to keep the telomeres shortening after each replication process. Otherwise, the living kind will grow in huge shape, as a result of a continuous dividing of non-dying cells. This is the effect of the cancer.

According to the above discussion, it is believed that cancer can be not counted as a negative agent, and it is for this matter, the cancer can be identified as:

A fundamental inherent mechanism which is created to eliminate severe damage to the vital process of the cells' performance, while the side effect of the cancer function is of great danger to the whole body.

If the above explanations are true for the cause of the cancer existence, then, it is believed that, there should be a certain method as a logical strategy to be followed for the possible cure of the cancer.

This should be depending on the fundamental points to be arising, as the following:

a- Some types of cancers are occurring many time in a person's life, [but] their effect is ended after short duration of time, without leaving any side effect.

This is performed when a damage or disease,, [which may cause the cancer], is cured, - but not the cancer itself-, by the body's immune or by a reasonable medical treatment. Where, there is no need for the cancer phase to be active for healing, i.e. the cancer will not continue to be existed.

b- The occurrence of the cancer is when a cell is severely affected by carcinogen factors as radioactive, highly concentrated of chemicals, etc...

It is, then, really dangerous to apply the same methods for the aiming to treat the cancer. This means that to cure the cancer, it is using similar methods to those which are causing it. It is believed that this is a case as if trying to eliminate the cancer, but by forming another phase of the onset of the cancer itself.

Therefore, the essential matter is that, the activity of the cancer will remain functioning if the treatment is associated with the application of the same traditional treatment methods, [hard treatments].

Accordingly, the cancer can be cured by applying a new strategy, concerning with the natural mechanism which is created the cancer itself. This strategy is, believed to be, as the following, in section 6 :

\section{Suggestion Strategic Method to Treat the Cancer}

Concerning with the above discussion, it is important to apply the following suggestion points:

A. Initially, it must be fully investigating the history of patient's diseases, to understand all diseases before the onset of the cancer, in particular the disease occurred in the time just preceding the cancer attack.
That is to fully justify what were the kind of previous diseases which may cause the cancer.

B. The emphasize is, then, concentrating only to fully cure from these previous diseases. This means that, in this step it should be entirely ignored the existence of the cancer and only to draw the attention to treat and cure these previous diseases.

C. It must be treated the immediate area surrounding the effected cancerous tissue to prevent any sort of diseases, or any possibility of the cancerous cells overcoming them.

D. The treatment, to the previous diseases, should be comprehensive and bases on the followings:

i). using the simplest materials and components when applying chemical or surgical methods, and

ii). Neither to use any form of radiation, hard surgery or strong chemicals, nor to expose (or subject) the effected tissue to what may damage it.

E. Apply a certain strength and direction of a magnetic field to treat the cancer cells, where the induced magnetic action is reducing the length of the telomere of the daughter DNA during the replication [5].

When the body is fully cured from the previous disease(s), or any dangerous factors, it is believed that, the body [or the effected tissue] is, now, in stable and healthy conditions, where there will be no need for extra treatment. Then the onset of the cancer is stopped.

It is according to the fact that, when people are affected by the same sort of disease, some of them are not liable to contract the cancer, but the others are liable, then:

In the first case it is the strength of the body, [by the immune system], that cures it from the previous disease. While in the second case the immune system of the body was unable to combat the other ailment, hence the consequences were cancerous attack.

This confirms the believe that, if it can be eliminated the previous factors causing the cancer, then it is no longer for the cancer to be existed. Finally, treating and cure the previous diseases are assumed to be a fundamental method to keep the cancer out.

The outcome of applying this strategy would be believed to show a great reduction in the number of the death rate among the cancer patients.

\section{Conclusion}

The essential reason for the onset of the cancer on cells, is to eliminate the severe action against the vital process of the cell's function. This means that the cancer has to be strategically understood in different manner than that of the traditional way. This understanding should be associated with the treatment method, which is applied to heal the body from the cancer itself.

The successful method of healing from cancer may occurred by full treatment method which is applied against the factors that were causing the cancer. It is not by direct treatment applied to the cancer itself. 
Treatments by applying the traditional methods are believed to be danger. They are the methods of trying to treat the cancer, but, truly, they are helping to cause the onset of the cancer itself.

\section{References}

[1] Little JB (2000). "Chapter 14: Ionizing Radiation". In Kufe DW, Pollock RE, Weichselbaum RR, Bast RC Jr, Gansler TS, Holland JF, Frei E III. Cancer medicine (6th ed.). Hamilton, Ont: B.C. Decker. ISBN 1-55009-113-1.

[2] Anand P, Kunnumakkara AB, Kunnumakara AB, Sundaram C, Harikumar KB, Tharakan ST, Lai OS, Sung B, Aggarwal BB (September 2008). "Cancer is a preventable disease that requires major lifestyle changes". Pharm. Res. 25 (9): 2097 116.

[3] Cancer Research UK : Cancer Help UK". Retrieved 11 May
2012.

[4] Blackburn, E.H. (2000), Telomere states and cell fates, Nature 408, 53-56.

[5] Rojeab, Adnan Yousif. The Processes of DNA Replication and the Shortening of the Telomere are Influenced by the Action of the Magnetic Field. International Journal of Genetics and Genomics. Vol. 2, No. 6, 2014, pp. 114-120. doi: 10.11648/j.ijgg.20140206.13.

[6] Kinzler, Kenneth W.; Vogelstein, Bert (2002). "Introduction". The genetic basis of human cancer (2nd, illustrated, revised ed.). New York: McGraw-Hill, Medical Pub. Division. p. 5. ISBN 978-0-07-137050-9.

[7] Kushi LH, Doyle C, McCullough M, et al. (2012). "American Cancer Society Guidelines on nutrition and physical activity for cancer prevention: reducing the risk of cancer with healthy food choices and physical activity". CA Cancer J Clin 62 (1): 30-67. 